\title{
Research on Esplanade-Cleaning Robot's Target Recognition
}

\author{
Jihao Li Ying Liu \\ Mechatronic Engineering Department \\ University of Science \& Technology Beijing \\ Beijing, China
}

\author{
Youyu Fan \\ Institute of Mechanics \\ Chinese Academy of Sciences \\ Beijing, China
}

\begin{abstract}
A kind of high-efficient target recognition algorithm has been worked out, which is on the basis of developing a esplanade-cleaning robotic prototype with a visual function. Based on the difference in value and target tricolor ratio, the robot can accurately identify the target garbage within its vision quickly. By the least squares method fitting that the target range, robot can accurately drive to the target position and control its manipulator to pick up the target rubbish. This algorithm could make the cleaning-robots working in outdoor plaza independently and without blindness, which can not only improve the efficiency, but also save energy greatly.
\end{abstract}

Keywords- Cleaning-robots; Distance measuring; Least-square method; Target recognition; Tricolor characteristics

\section{INTRODUCTION}

To improve the efficiency of the environmental cleaningrobots and reduce costs, developing the environmental cleaning robots with visual function in [1] has become an inevitable trend. Except obtaining essential information such as obstacles and boundaries etc, traditional cleaning robot in [2] cannot basically judge whether there is rubbish and the specific garbage location. Thus it requires uninterrupted complete scope of carpet cleaning, which wastes energy greatly. Compared with traditional cleaning robots, the environmental cleaning robots with visual function can get more information from the outside, which has important meaning to the intelligence of cleaning-robots.

During the work, cleaning robots need to move continually, and the rubbish may be also moving, so the target recognition process must be a real-time one, and needs certain tracking abilities for the mobile garbage. At present, the common recognition algorithm is using grayscale image and complex algorithms to match the object in [3], the identification speed is low, and demanding high computational speed. Thus ,one kind of new algorithm, which can be performed quickly in the upper computer, is necessary to be used to solve the problems.

A kind of square-cleaning robot has been developed, which can work in the flat ground outdoor. This kind of robots with visual function can search independently and clean the rubbish. To identify the target rapidly and accurately, a target recognition algorithm has been proposed, which is based on the target tricolor characteristics and the least-square method, so that the robots can clean rubbish efficiently and autonomously.

\section{ESPLANADE-CLEANING ROBOT}

The traditional cleaning robots usually use aspiration type working principle, so they are helpless to some slightly bigger garbage. In order to overcome the cleaning robots' blind carpet cleaning and drawbacks mentioned above, according to the functional requirements such as the obstacle avoidance, target recognition, distance calculations, wireless communications, manipulator grab etc, the square cleaning robotic prototype with visual function as shown in Fig.1 has been developed, whose system frame as Fig.2 shows. The robot is mainly composed of driving system, manipulator by grabbing system, electronic control system and visual system structure.

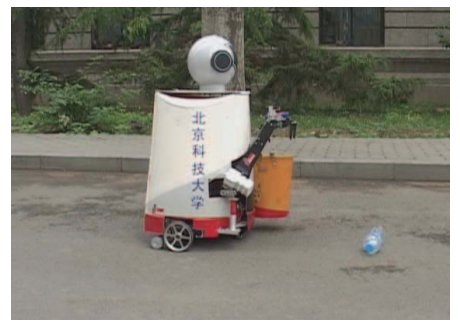

Figure 1. Prototype of the robot

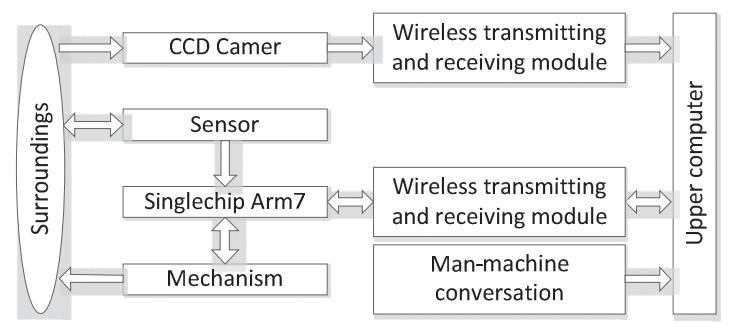

Figure 2. System frame

The robot works as below: the robot captures images with the camer, delives the images to PC computer via wireless transmission module, and activates target recognition algorithm to recognize the target within the vision. If identified the goal, the robot will calculate the distance of the target relative to the robot itself. Fig. 3 shows its working principle diagram. 


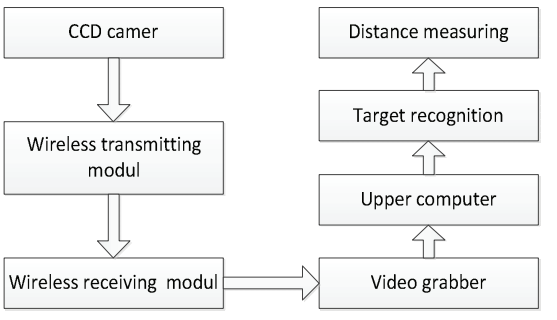

Figure 3. Working principle diagram

\section{TARGET RECOGNITION}

\section{A. Target Recognition Algorithm}

Based on gray images, the traditional target recognition algorithm using threshold segmentation, contour extraction, object matching is hard to meet the robot's real-time processing image requirements. An effective simplified algorithm has been proposed to reduce the time of target recognition significantly, which is one of the bottleneck problems. This algorithm based on target's tricolor (blue as B, green as G and red as R) characteristics in [4]. Using the mineral water bottles on the square as an example, the robot's recognition process is shown in Fig.4.

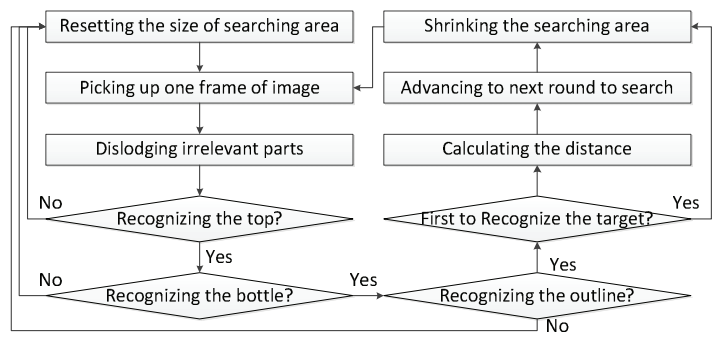

Figure 4. Robot's recognition process

Before activating algorithm, the robot needs to collect the colors of the object with obvious colorful feature, calculate the standard color characteristic $\mathrm{ST}_{\mathrm{k}}, \mathrm{k}$ as $1,2,3 \ldots$ in every part. Robot judges eligible goals of $\mathrm{ST}_{1} \cap \mathrm{ST}_{2} \cap \ldots \cap \mathrm{ST}_{\mathrm{k}}$ as rubbish.

\section{1) Setting standards $S T_{k}$}

The mineral water bottles on the square are used as an example to illustrate the target identification process. Mineral water bottles have two main parts with obvious colorful feature: the cap and the bottle. Only the object fulfills the cap standard $\mathrm{ST}_{1}$ and the bottle standard $\mathrm{ST}_{2}$ at the same time that the object is considered to be a mineral water bottle.

A group of RGB data $I=\left\{B_{i}, G_{i}, R_{i} \mid i=1,2,3 \ldots n\right\}$ of the cap can be got from multiple images. Light intensity in the external environment is changing constantly, but in terms of some kind of material's reflective properties to one color, its difference between RGB data (B-G, B-R, G-R) and the ratio $(\mathrm{B} / \mathrm{G}, \mathrm{B} / \mathrm{R}, \mathrm{G} / \mathrm{R})$ only fluctuates in a relatively fixed range. By combining the date I differently, dates can be got as below:

$$
X=\left\{Y_{p} \mid p \in[1,6]\right\}
$$

With:

$$
\begin{aligned}
& Y_{1}=\left\{y_{1 i}=B_{i}-G_{i} \mid i=1,2,3 \cdots n\right\} \\
& Y_{2}=\left\{y_{2 i}=B_{i}-R_{i} \mid i=1,2,3 \cdots n\right\} \\
& Y_{3}=\left\{y_{3 i}=G_{i}-R_{i} \mid i=1,2,3 \cdots n\right\} \\
& Y_{4}=\left\{y_{4 i}=B_{i} / G_{i} \mid i=1,2,3 \cdots n\right\} \\
& Y_{5}=\left\{y_{5 i}=B_{i} / R_{i} \mid i=1,2,3 \cdots n\right\} \\
& Y_{6}=\left\{y_{6 i}=G_{i} / R_{i} \mid i=1,2,3 \cdots n\right\}
\end{aligned}
$$

Calculating the average $\bar{X}$ and covariance $\delta$ according to the data $X$ :

$$
\bar{X}=\left\{\overline{Y_{p}}=\sum_{i=1}^{n} y_{p i} / n \mid p \in[1,6]\right\}
$$

$$
\delta=\left\{\delta_{p}=\sqrt{\sum_{i=1}^{n}\left(y_{p i}-\overline{Y_{p}}\right)^{2} / n} \mid p \in[1,6]\right\}
$$

Setting $S=[\bar{X}-\delta, \bar{X}+\delta]$,if $y_{p i} \notin S$, the data $\mathrm{y}_{\mathrm{pi}}$ will be thought wrong. Aiming at suppressing the influence of wrong data $\mathrm{y}_{\mathrm{pi}}$ without reducing the capacity of the sample, $\mathrm{y}_{\mathrm{pi}}$ is treated as the new average of the sample;if $y_{p i} \in S, \mathrm{y}_{\mathrm{pi}}$ will remain unchanged.

$$
y_{p i}^{\prime}=\left\{\begin{array}{l}
\frac{m \overline{Y_{p}}-y_{p i}}{m-1}, y_{p i} \notin S \\
y_{p i}, y_{p i} \in S
\end{array}\right.
$$

Recalculating according to $y_{p i}^{\prime}$ :

$$
\overline{X^{\prime}}=\left\{\overline{Y_{p}^{\prime}} \mid p \in[1,6]\right\}
$$

$$
\delta^{\prime}=\left\{\delta_{p}^{\prime} \mid p \in[1,6]\right\}
$$

$S T_{1}=\left[\overline{X^{\prime}}-\delta^{\prime}, \overline{X^{\prime}}+\delta^{\prime}\right]$ is the standard about the top of the mineral water bottle as $\mathrm{ST}_{1}$. And the standard $\mathrm{ST}_{2}$ about the body of the bottle can be educed according to the above method.The cleaning Robot can find out the target rubbish through the image by the standard $\mathrm{ST}_{1} \& \mathrm{ST}_{2}$.

2) Precessing the image according to $S T_{k}$ 
Defining the image's width as w,and image's height as $\mathrm{h}$,scaning the image point wise from the up to down of the image and then Calculating the error and ratio among the three primary colors of every point in turn,the equation as follows can be educed through (1).

$$
X=\left\{y_{p i} \mid p \in[1,6], i \in[1, w \times h]\right\}
$$

Ordering $\quad Q_{i}=\left\{\begin{array}{l}1, y_{p i} \in S T_{1}, p \in[1,6] \\ 0, y_{p i} \notin S T_{1}, p \in[1,6]\end{array}\right.$

The source image(Fig.5a) processed by the standard shows as the Fig.5b. When $\mathrm{Q}_{\mathrm{i}}$ equals 1,the colour of the point will change into black; When $\mathrm{Q}_{\mathrm{i}}$ equals 0 , the colour of the point will change into white.

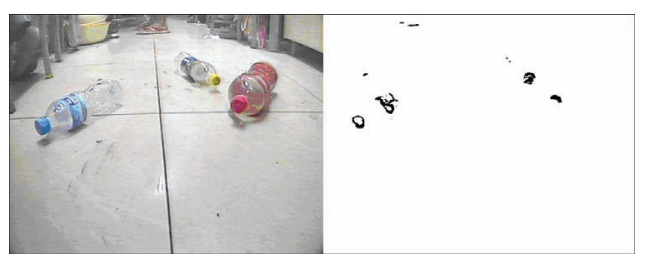

Figure 5. Source image and Processed by standatd $\mathrm{ST}_{1}$

Because not all targets with the similar kinds of colours can be told apart through the standard $\mathrm{ST}_{1}$, the standard $\mathrm{ST}_{2}$ is necessary to be used to make a further judgement. In consideration of that the body of mineral water bottle must lay beside the top,only the points in the rectangular region, whose center is just the position of top and size is $\mathrm{M} \times \mathrm{M}$, need to be processed by the standard $\mathrm{ST}_{2}$.

The big rectangular region is divided into several small rectanglular region,whose edge length is $\mathrm{M} / \mathrm{f}$.Every small rectangle is marked in turn as $\mathrm{R}_{1}, \mathrm{R}_{2}, \cdots, \mathrm{R}_{\mathrm{k}}, \cdots, \mathrm{R}_{\mathrm{fxf}}$. And the average of the sum of the difference or ratio among three primary colours of the points in the rectanglular region $R_{k}$ is treated as $\mathrm{A}_{\mathrm{pk}}$.

$$
A_{p k}=\sum y_{p i} / f^{2}, Q_{i} \in R_{k}, p \in[1,6]
$$

Ordering $\quad T_{k}=\left\{\begin{array}{l}1, A_{p k} \in S T_{2}, p \in[1,6] \\ 0, A_{p k} \notin S T_{2}, p \in[1,6]\end{array}\right.$

If $T=\sum_{k=1}^{f x f} T_{k} / f^{2} \geq \frac{1}{3}$, the location of the top will be treated as the position of the bottle.The image precessed by the standard $\mathrm{ST}_{1}$ and $\mathrm{ST}_{2}$ is showed as the Fig.6a.

In consideration of the disturbances from the transmission over radio, a second judgement is necessary for corroboration after the first judgement by standard ST1 \& ST2 .To make the calculating speed faster and to reduce most of the influence of disturbances, a searching region(Fig.6b) has been built.The second judgement only needs to be performed in this region. If the first judgement is similar to the next judgement, the target will be treated as mineral water bottle;if not,a new round of judgement will be installed.

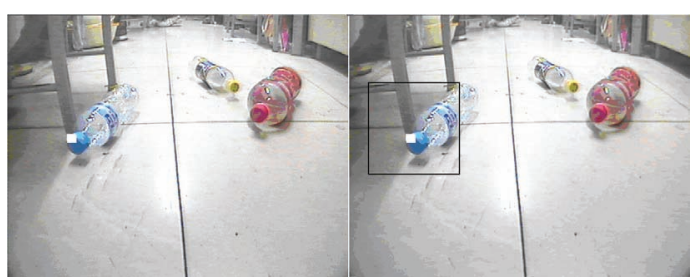

Figure 6. Position of the bottle and searching region

\section{B. Calculating the Distance of Target Rubbish}

The geometric model of camer is usually treated as pinhole cameramodel in [5],through which the connection of one-toone mapping of world cordinates and image cordinates can be founded.But this kind of connection of one-to-one mapping, which is about two planes and based on single camer,cannot be used to calculate the distance between the robot and target which is not on ground. Because it's hard to make sure that the position (white block in Fig. 6a) is close to ground, the least square method is choosed to calculate the distance to weaken the influence of errors.

\section{1) Calculating the distance at y-axis}

According to the preliminarily setted distance, nine object points at the midline of the image plane have been choosed to calculate the distance at y-axis. The letter y has been treated as the ordinates of points in the image plane,and the word Posy,whose unit is centimeter, as actual distance at y-axis on the ground. Table I shows the data about the object points.

TABLE I. OBJECT POINTS AND ACTUAL DISTANCE AT Y-AXIS

\begin{tabular}{|c|c|c||c|c|c|}
\hline No. & $\mathbf{y}$ & Posy & No. & y & Posy \\
\hline 1 & 37 & 20 & 6 & 408 & 150 \\
\hline 2 & 145 & 30 & 7 & 425 & 200 \\
\hline 3 & 214 & 40 & 8 & 438 & 250 \\
\hline 4 & 265 & 50 & 9 & 446 & 300 \\
\hline 5 & 370 & 100 & - & - & - \\
\hline
\end{tabular}

To ensure the correctness and high precision, dividing the data into three parts and processing the data in the least square method educes (10):

$$
\text { Posy }=\left\{\begin{array}{l}
0.0003 \mathrm{y}^{2}+0.0302 \mathrm{y}+18.456,0 \leq y \leq 250 \\
0.0034 \mathrm{y}^{2}-1.5676 \mathrm{y}+223,250<y \leq 370 \\
0.0353 \mathrm{y}^{2}-26.261 \mathrm{y}+4980.9,370<y<480
\end{array}\right.
$$

Based on (10), any point $(x, y)$ in the image plane maps the actual ground point(Posx,Posy). Fig.7 shows the fitting curves. 


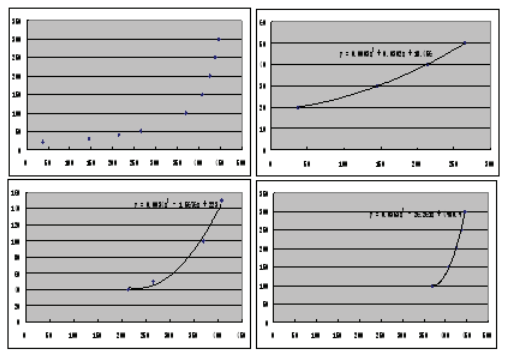

Figure 7. Fitting curves at $y$-axis

\section{2) Calculating the distance at $x$-axis}

Four pairs of object points in the image plane, which are symmetrical about y-axis, have been choosed with an interval of 50 centimeters. The data about the points have been filled in the Table II.Calculating the average of every pair of object point eduals new data,with which curve(Fig. $8 \mathrm{~b})$ is fitted in the least square method. If one point's horizontal coordinates is $\mathrm{x}$ in the image plane and vertical coordinates is Posy on the ground, its horizontal ordinate Posx, whose unit is centimeter, can be calculated by (11).

$$
\operatorname{Pos} x=\frac{50(x-320)}{0.0042 \text { Posy }^{2}-2.31 \text { Posy }+417.25}
$$

TABLE II. OBJECT POINTS AND ACTUAL DISTANCE AT X-AXIS

\begin{tabular}{|c|c|c|c|c|}
\hline No. & $\mathbf{x}$ & $\mathbf{y}$ & Posx & Posy \\
\hline 1 & 229 & 358 & & 100 \\
\hline 2 & 163 & 401 & \multirow{5}{*}{50} & 150 \\
\hline 3 & 126 & 423 & & 200 \\
\hline 4 & 147 & 437 & & 250 \\
\hline
\end{tabular}

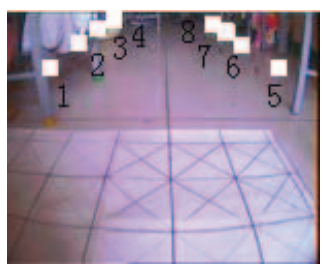

a) Object Points

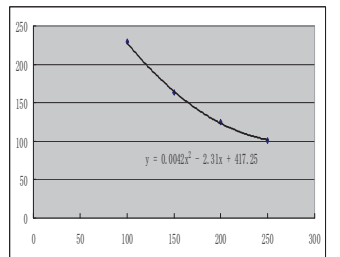

b) Fitting Curve
Figure 8. Object points and fitting curve at $\mathrm{x}$-axis

\section{RESEARCHES OF EXPERIMENT}

As the cleaning robot recognises several different kinds of target rubbish and calculates the distance between the robot and target rubbish rightly, it can reach to the right location where the target rubbish locates. The experiment has verified the correctness and availability of the method of target recognition. In view of existing situation of the cleaning robot,which will use its mechanical arm to pick up the target rubbish,numerous experiments about robot picking mineral water bottles have been performed.
The surroundings of experiment is flat blacktop and the target rubbish is the mineral water bottles on the gound. Fig.9 shows the process that the cleaning robot picks rubbish. The experiment shows that the cleaning robot can work better with the vision system with the method of target recognition. If higher performance mechanical arm can be used and the database of diffternt kinds of rubbish gets extended, the cleaning robot will have a better ability to pick more varieties of rubbish and the cleaning effect will be more ideal

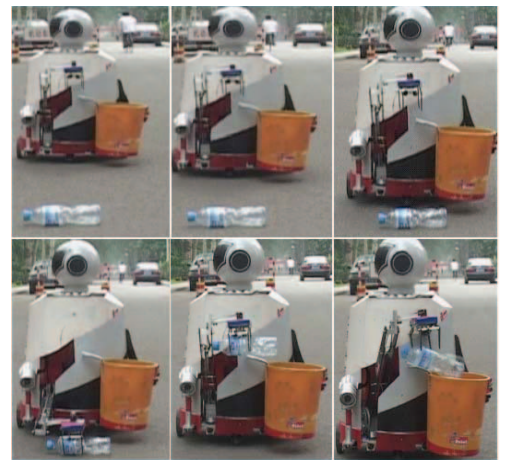

Figure 9. Experiment of picking

\section{CONCLUSION}

After analyzing the difference and ratio among the three primary colours, One new method about image processing based on the characters of tricolor has been brought forward innovatively. Banding with the highly reliable method to calculate the distance between the robot and the target rubbish based on least square method,one method of target recognition, which is true of cleaning robot, has been raised.With this metod, only when the cleaning robot finds out and reaches to the target rubbish, it initializes the program to pick the rubbish instead of working blindly.Thus a cleaning robot with the ability of recognizing targets can save much more energy source and lower costs than one without the ability.That the reality of this method of target recognition lays the foundation for the further research of cleaning robot with vision. Simultaneously, in some dangerous or complex cases, it can be used to do some operations instead of human beings.

\section{REFERENCES}

[1] Xueqiang Xi,Runsheng Wang,Knowledge and its Representation of a Computer vision Model. Journal of Computer Engineering and applying,November 2000,pp.45-91. (In Chinese).

[2] Dingzhong Tan, Research Status and Development of Cleaning Robot. Journal of Mechanical Engineers, June 2004 , pp.9-11 (In Chinese).

[3] Mingdong Xue, Li Guo, A New Method of Image Recognition. Journal of Computer Engineering, vol.31,May 2005, pp.173-175 (In Chinese).

[4] Sheng Huang,Hua Zheng, A Method for Extracting RGB-Color-Regions from Images,vol.31,September 2009, pp.48 (In Chinese).

[5] Nicolas Alvertos, Dragana Brzakovic, Rafael C. Gonzalez, Camera Geometries for Image Matching in 3-D Machine Vision. IEEE Transactions on Pattern Analysis and Machine intelligence, VOL. 11,NO. 9,September 1989 . 